\title{
Involutions on standard Young tableaux and divisors on metric graphs
}

\author{
Rohit Agrawal \\ University of Minnesota \\ Minneapolis, Minnesota, U.S.A. \\ agraw025@umn . edu \\ Vladimir Sotirov \\ University of Wisconsin, Madison \\ Madison, Wisconson, U.S.A. \\ sotirov@math.wisc.edu
}

\author{
Gregg Musiker \\ University of Minnesota \\ Minneapolis, Minnesota, U.S.A. \\ musiker@math . umn . edu \\ Fan Wei \\ Massachusetts Institute of Technology \\ Cambridge, Massachusetts, U.S.A. \\ fanwei@alum.mit.edu
}

Submitted: Aug 14, 2012; Accepted: Aug 27, 2013; Published: Sep 6, 2013

Mathematics Subject Classifications: 05C57, 06A07, 14N10, 14 T05

\begin{abstract}
We elaborate upon a bijection discovered by Cools, Draisma, Payne, and Robeva (2012) between the set of rectangular standard Young tableaux and the set of equivalence classes of chip configurations on certain metric graphs under the relation of linear equivalence. We present an explicit formula for computing the $v_{0}$-reduced divisors (representatives of the equivalence classes) associated to given tableaux, and use this formula to prove (i) evacuation of tableaux corresponds (under the bijection) to reflecting the metric graph, and (ii) conjugation of the tableaux corresponds to taking the Riemann-Roch dual of the divisor.
\end{abstract}

Keywords: Metric graphs; Tropical geometry; Divisors on graphs; Chip-firing; Young tableaux; Evacuation

\section{Introduction}

In [4], Baker reduces the Brill-Noether Theorem, which concerns linear equivalence classes of divisors on a normal smooth projective curve $X$ of genus $g$, to an analogous statement regarding linear equivalence classes of divisors on certain abstract tropical curves. In [5], Cools, Draisma, Payne, and Robeva then use this reduction to provide a tropical proof of the Brill-Noether Theorem valid over algebraically closed fields of any characteristic. 
The abstract tropical curves in question are compact metric graphs $\Gamma_{g}$, illustrated in Figure 1, consisting of a chain of $g$ concatenated loops and designated vertices $\left\{v_{0}, \ldots, v_{g}\right\}$ such that the edge lengths $\left(\ell_{i}, m_{i}\right)$ satisfy a certain genericity condition.

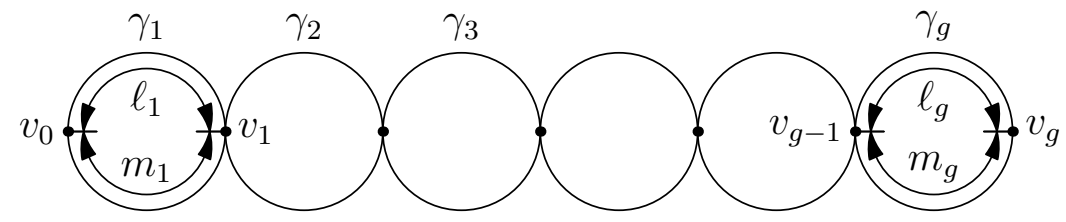

Figure 1: A metric graph $\Gamma_{g}$ together with its designated vertices and edge lengths.

In the course of their proof, Cools et al. find a bijection from rectangular standard Young tableaux to the linear equivalence classes of rank $r$ degree $d$ divisors on $\Gamma_{g}$ that satisfy $g=(g-d+r)(r+1)$. This bijection raises the question of how natural operations on rectangular standard Young tableaux, such as evacuation and conjugation (i.e. transpose), translate for divisors on generic metric graphs $\Gamma_{g}$. In this paper, we prove that evacuation reflects the generic graph $\Gamma_{g}$ and that conjugation exchanges a divisor $c$ with its RiemannRoch dual $K-c$. Note that in this paper we only focus on the bijection aspect of the deep result of [5], and leave other applications for future work.

In Section 2 we review both the background material necessary to understand the bijection due to Cools et al. and the bijection itself. Given our combinatorial goal, our presentation of their combinatorial results differs from the one in their paper. In particular, we reformulate their theorem [5, Theorem 1.4] as a specification of an algorithm for computing ranks of divisors on the graphs $\Gamma_{g}$. In this formulation, their bijection follows as a property of that algorithm.

In Section 3 we state and prove our first result, Theorem 24, equating evacuation and reflection, by providing formulas for the bijection and its compositions with these two operations. In Section 4, we then discuss our second result, Theorem 39, equating conjugation of tableaux to the map $c \mapsto K-c$ on divisor classes.

Before reading Sections 3 and 4, the reader familiar with the combinatorics of [5] will need to read only Lemma 9 and Subsection 2.3, in which we introduce the notation that we use to prove our main results.

\section{The bijection $\phi$ of Cools et al.}

In this section we will review the theory of divisors on metric graphs, and the results that go into the derivation of the bijection of Cools et al. For a more detailed exposition on metric graphs, we refer the reader to [8,2], which our exposition partially follows. For an exposition of the tropical proof of the Brill-Noether theorem, we recommend the original paper [5]. 


\subsection{Basic notions of compact metric graphs and their divisors}

In general, a metric graph $\Gamma$ is a complete metric space such that every point $x \in \Gamma$ is of some valence $n \in \mathbb{N}$, meaning that there exists an $\epsilon$-ball centered at $x$ isometric to the star-shaped metric subspace $V(n, \epsilon)=\left\{t e^{i \frac{k n}{2 \pi}}: 0 \leqslant t<\epsilon, k \in \mathbb{Z}\right\} \subset \mathbb{C}$ endowed with the path metric.

A model of a compact metric graph $\Gamma$ is a finite weighted multigraph without loops $G$ such that its vertex set $V$, which is a finite subset of $\Gamma$, satisfies the property that the connected components of $\Gamma \backslash V$ are isometric to open intervals. The weighted edge multiset $E$ of $G$ is uniquely determined by the set of $G$ 's vertices as follows. For each connected component of $\Gamma \backslash V$ whose boundary points in $\Gamma$ are precisely $\{v, w\} \subset V$, we add an edge between $v$ and $w$ of weight equal to the length of the isometric open interval ${ }^{1}$. In particular, the designated vertices $\left\{v_{0}, \ldots, v_{n}\right\}$ give one model for the compact metric graph $\Gamma_{g}$ (illustrated in Figure 1), but that is not the only possible model, as illustrated in Figure 2.
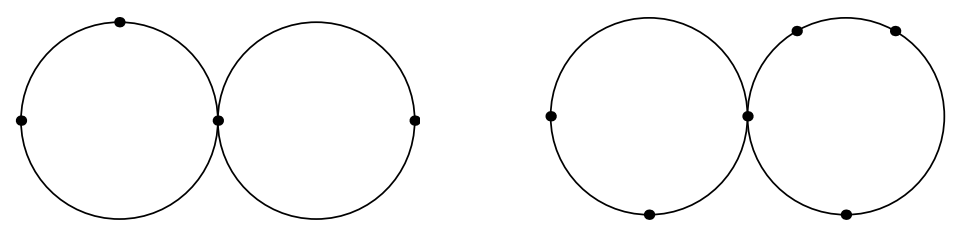

Figure 2: Two other models for the compact metric graph $\Gamma_{2}$.

The genus $g$ of a compact metric graph $\Gamma$ can be defined to be $|E|-|V|+k$ where $(V, E)=G$ is a model of $\Gamma$ and $k$ is the number of connected components. It is easy to show that given two models $G_{1}=\left(V_{1}, E_{1}\right)$ and $G_{2}=\left(V_{2}, E_{2}\right)$, both give the same genus as the model $G_{3}=\left(V_{1} \cup V_{2}, E_{3}\right)$. In particular, the genus of the graphs $\Gamma_{g}$ is precisely $g$.

A divisor on a compact metric graph $\Gamma$ is an element of the free abelian group $\operatorname{Div}(\Gamma)$ generated by the points of $\Gamma$. There is nothing deep about the group of divisors; the deep analogy between the theory of divisors on compact metric graphs and the theory of divisors on Riemann surfaces comes from the definitions of rational functions on $\Gamma$, their orders at points on $\Gamma$, and the consequent notion of equivalence of divisors, which is strong enough for an analogue of the Riemann-Roch theorem to hold (the so-called Tropical Riemann-Roch Theorem).

A rational function $f$ on a compact metric graph $\Gamma$ is a continuous function $f: \Gamma \rightarrow \mathbb{R}$ that is piecewise linear with integer slopes in the following sense: there exists a model $G_{f}$ of $\Gamma$ such that the restriction of $f$ to each edge is a linear function with integer slope. We set the order of $f$ at $x$, $\operatorname{ord}_{x}(f)$, to be 0 if $x$ is not a vertex of $G_{f}$, and otherwise we set $\operatorname{ord}_{x}(f)$ to be the sum of the outgoing slopes along edges coming out of $x$. Note that $\operatorname{ord}_{x}(f)$ is non-zero for only finitely many points $x$ and that $\operatorname{ord}_{x}: \operatorname{Div}(\Gamma) \rightarrow \mathbb{Z}$ is a homomorphism as $\operatorname{ord}_{x}(f+g)=\operatorname{ord}_{x}(f)+\operatorname{ord}_{x}(g)$.

\footnotetext{
${ }^{1}$ By abuse of notation, we will identify the edges of $G$ with the closed intervals that are isometric to the closures of the connected components of $\Gamma \backslash V$. By disallowing models which contain loops, we ensure that these closures are still line segments.
} 
Two divisors $c$ and $c^{\prime}$ are said to be equivalent if $c-c^{\prime}=\sum_{x \in \Gamma} \operatorname{ord}_{x}(f)(x)$ for some rational function $f$. The divisors of the form $\sum_{x \in \Gamma} \operatorname{ord}_{x}(f)$ form an abelian group called the group of principal divisors. The quotient of $\operatorname{Div}(\Gamma)$ by the group of principal divisors is denoted by $\operatorname{Pic}(\Gamma)$ and consists of the equivalence classes of divisors under the above equivalence relation.

Given a divisor $c$, the degree of $c$ is defined to be $\operatorname{deg}(c)=\sum_{x \in \Gamma} c(x)$. The degree is invariant under equivalence, as any principal divisor has degree 0 . Hence every element of $\operatorname{Pic}(\Gamma)$ has a well-defined degree as well. Given an integer $d$, we define $\operatorname{Pic}^{\leqslant d}(\Gamma)$ to be the subset of $\operatorname{Pic}(\Gamma)$ with degree at most $d$.

A divisor $e=\sum_{i=1}^{n} a_{i}\left(x_{i}\right)$ is said to be effective if $a_{i} \geqslant 0$ for all $1 \leqslant i \leqslant n$. A divisor $c$ that is not equivalent to an effective divisor is said to have $\operatorname{rank} r(c)=-1$. A divisor $c$ that is equivalent to an effective divisor is said to have $\operatorname{rank} r(c)=r$ if $r$ is the largest number such that for every effective divisor $e$ of degree $r$, the divisor $c-e$ is equivalent to an effective divisor. Note that in particular $r(c) \leqslant \min \{-1, \operatorname{deg}(c)\}$ since if $c-e$ has negative degree, then it cannot be equivalent to an effective divisor.

The interest in this notion of rank stems from the fact that it is invariant under equivalence of divisors and, more importantly, that it satisfies an analogue of the RiemannRoch theorem, first proven for finite graphs by Baker and Norine in [3] and subsequently generalized for metric graphs and tropical curves independently by Gathmann and Kerber in [7] and Mikhalkin and Zharkov in [10].

Theorem 1 (Tropical Riemann-Roch). Suppose that $c \in \operatorname{Div}(\Gamma)$ is a divisor on a compact metric graph. Define the canonical divisor $K$ on $\Gamma$ by $K=\sum_{x \in \Gamma}(\operatorname{val}(x)-2)(x)$. Then we have:

$$
r(c)-r(K-c)=\operatorname{deg}(c)+1-g
$$

where $g$ is the genus of $\Gamma$.

\subsection{The graphs $\Gamma_{g}$, and their $v_{i}$-reduced divisors}

In their paper, Cools et al. reduce the verification of the Brill-Noether theorem to an analysis of the ranks of divisors on members of the following family of genus $g$ graphs.

Definition 2. The compact metric graphs $\Gamma_{g}$ consist of $g$ circles $\left\{\gamma_{i}\right\}_{1 \leqslant i \leqslant g}$ of circumferences $\left\{\ell_{i}+m_{i}\right\}$ concatenated together in such a way that there exists a model with vertices $\left\{v_{i}\right\}_{0 \leqslant i \leqslant g}$ so that for every $1 \leqslant i \leqslant g, v_{i-1}$ and $v_{i}$ are designated vertices of $\gamma_{i}$ and the two edges of $\gamma_{i}$ joining $v_{i}$ and $v_{i-1}$ have lengths $\ell_{i}$ and $m_{i}$. The metric on $\Gamma_{g}$ is the path-length metric. See Figure 1 for an illustration.

In particular, Cools et al. are interested in computing the rank of an arbitrary divisor on $\Gamma_{g}$. In the case where the divisor has degree greater than $2 g-2$, there is a simple answer using the tropical Riemann-Roch theorem, agreeing with the answer in the case of algebraic curves. 
Proposition 3. Any divisor $c$ on the compact metric graph $\Gamma_{g}$ of degree greater than $2 g-2$ has rank $\operatorname{deg}(c)-g$.

In the case where the divisor has degree at most $2 g-2$, the computation of the rank becomes extremely difficult in general. For graphs $\Gamma_{g}$ satisfying a certain genericity condition, Cools et al. give an elementary algorithm for performing the computation. In this subsection we describe first the input to the algorithm, which consists of certain divisors known as $v$-reduced divisors, and second the genericity condition that the graphs $\Gamma_{g}$ must satisfy for the algorithm to work correctly.

The notion of $v$-reduced divisors for graphs was first introduced by Baker and Norine in [3] as a slight variant of the notion of $G$-parking functions introduced by Postnikov and Shapiro in [12]. Their importance for the theory of divisors stems from the fact that they provide a system of representatives of the group $\operatorname{Pic}(\Gamma)$ of equivalence classes of divisors. Unfortunately, the language of divisors is somewhat clunky for describing the $v$-reduced divisors and their properties, so instead we consider a divisor $\sum_{i=1}^{n} a_{i}\left(x_{i}\right) \in \operatorname{Div}(\Gamma)$ to be the chip configuration that assigns to each point $x_{i}$ the respective amount of $a_{i}$ chips. From here onward, we will use the terms "chip configuration" and "divisor" interchangeably. In particular, by the degree and rank of a chip configuration we will mean the degree or rank of that divisor.

The following definition of $v$-reduced divisors for metric graphs is due to Luo [9].

Definition 4. Fix a point $v$ on a connected compact metric graph $\Gamma$. A chip configuration $c \in \operatorname{Div}(\Gamma)$ is called a $v$-reduced divisor if:

1. $c$ has a non-negative number of chips on every point except for possibly $v$;

2. for any closed connected subset $X \subset \Gamma$ not containing $v$, there exists a point $x$ in the boundary of $X$ such that the number of chips on $x$ is strictly smaller than the number of edges from $x$ to $\Gamma \backslash X$ (the number of edges joining $x$ to a point in $\Gamma \backslash X$ for any model $G$ of $\Gamma$ such that $x$ is a vertex in $G$ and $G$ restricts to a model of $X$ ).

In [9], Luo generalizes the so-called burning algorithm due to Dhar [6] for finding $v$ reduced divisors from the case of finite graphs to the case of metric graphs, and uses it to prove that for any $v$ in a connected compact metric graph $\Gamma$, the set of $v$-reduced divisors is a system of representatives for $\operatorname{Pic}(\Gamma)$.

Theorem 5 (Theorem 2.3 of [9]). Suppose that $\Gamma$ is a connected compact metric graph, and that $v$ is a designated point on $\Gamma$. Then every chip configuration $c \in \operatorname{Div}(\Gamma)$ is equivalent to exactly one $v$-reduced divisor.

Using Luo's generalization of Dhar's burning algorithm, which we will not describe, one can easily compute for any chip configuration $c$ on $\Gamma_{g}$ the $v_{i}$-reduced divisors equivalent to $c$. Since the rank of a chip configuration is invariant under equivalence, being able to determine the rank of $v_{i}$-reduced divisors is enough to determine the rank of any chip configuration. 
Cools et al. noticed that the $v_{i}$-reduced divisors on the graph $\Gamma_{g}$ have an elementary description which follows almost immediately from the definitions. This description in turn suggests a compact notation for the elements of $\operatorname{Pic}\left(\Gamma_{g}\right)$ once we identify them with $v_{0}$-reduced divisors.

Proposition 6 (Example 2.6 of [5]). The $v_{i}$-reduced divisors on $\Gamma_{g}$ are precisely those chip configurations $c \in \operatorname{Div}\left(\Gamma_{g}\right)$ for which:

1. every point different from $v_{i}$ has a non-negative number of chips;

2. each of the cut loops to the right of $v_{i}$ (given by $\gamma_{j} \backslash\left\{v_{j-1}\right\}$ for $j>i$ ) and to the left of $v_{i}$ (given by $\gamma_{j} \backslash\left\{v_{j}\right\}$ for $j \leqslant i$ ) contain at most 1 chip.

Example 7. Consider a compact metric graph $\Gamma_{6}$ with edge lengths $m_{1}=m_{2}=\cdots=$ $m_{6}=1$ and $\ell_{1}=\ell_{2}=\cdots=\ell_{6}=10$ (see Figure 1 ). The following, which we will use as a running example, illustrates a $v_{0}$-reduced divisor which has 2 chips on $v_{0}$ and at most 1 chip on every loop $\gamma_{i} \backslash\left\{v_{i-1}\right\}$ for $1 \leqslant i \leqslant g$. Note that the extra vertices shown in the first two loops are unnecessary, but help clarify the distances in this model.

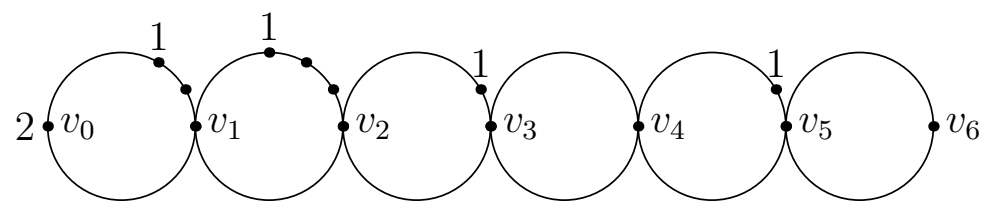

Definition 8. To every $v_{0}$-reduced divisor on $\Gamma_{g}$, and hence to every element of $\operatorname{Pic}\left(\Gamma_{g}\right)$, we associate sequences $\left(d_{0} ; x_{1}, x_{2}, \ldots, x_{g}\right)$ where $d_{0}$ is the number of chips on $v_{0}$, and $x_{i} \in \mathbb{R}$ a distance (modulo the circumference of the $i^{\text {th }}$ loop) from $v_{i-1}$ in the counter-clockwise direction of the single chip on $\gamma_{i} \backslash\left\{v_{i-1}\right\}$, with $x_{i}=0$ if there is no chip (regardless of the possible existence of chips on $\left.v_{i-1}\right)$. These sequences are unique up to the modular equivalences $x_{i} \bmod \left(\ell_{i}+m_{i}\right)$.

For Example 7 this sequence is $(2 ; 3,4,2,0,2,0)$.

Next, we describe the genericity condition that the graphs $\Gamma_{g}$ must satisfy for the algorithm to work correctly. The following lemma, which motivates genericity, is Example 2.1 in [5] and is crucial both for their algorithm and for the proof of our own Theorem 24.

Lemma 9 (Recentering $v_{i}$-reduced divisors on $\Gamma_{g}$ ). Let c be a $v_{i-1}$-reduced divisor. Suppose that $c$ has $k$ chips on $v_{i-1}$, and that the chip on $\gamma_{i} \backslash\left\{v_{i-1}\right\}$ is a counter-clockwise distance $x_{i}$ from $v_{i-1}$ (with $x_{i}=0$ if there is no chip).

Then $c$ is equivalent to the $v_{i}$-reduced divisor $c^{\prime}$ which agrees with c everywhere outside $\gamma_{i}$, and which restricts on $\gamma_{i}$ according to the following cases:

1. If $x_{i}=0$ and $k \geqslant 1$, then $c^{\prime}$ has $k-1$ chips on $v_{i}$ and one chip that is $(k-1) m_{i}$ away clockwise from $v_{i-1}$;

2. if $x_{i} \not \equiv(k+1) m_{i} \bmod \left(\ell_{i}+m_{i}\right)$ then $c^{\prime}$ has $k$ chips on $v_{i}$ plus a chip that is $k m_{i}-x_{i}$ away clockwise from $v_{i-1}$; 
3. if $x_{i} \equiv(k+1) m_{i} \bmod \left(\ell_{i}+m_{i}\right)$, then $c^{\prime}$ has $k+1$ chips on $v_{i-1}$.

Note that the first case can result in all $k$ chips being moved from $v_{i-1}$ to $v_{i}$ if and only if $(k-1) m_{i} \equiv \ell_{i} \equiv-m_{i} \bmod \left(\ell_{i}+m_{i}\right)$, which is the same as requiring that $k m_{i}$ is an integer multiple of $\ell_{i}+m_{i}$, i.e. that $\frac{\ell_{i}}{m_{i}}=\frac{k-n}{n}$ for some positive integer $n$. If $\operatorname{deg}(c) \leqslant 2 g-2$, however, then certainly $k \leqslant 2 g-2$, so this can only happen if $\ell_{i} / m_{i}$ can be written as the ratio of two integers with sum at most $2 g-2$. Thus, the notion of genericity that we define below ensures that the first case of the lemma never results in all $k$ chips being moved from $v_{i-1}$ to $v_{i}$.

Definition 10. We say that $\Gamma_{g}$ is generic if none of $\ell_{i} / m_{i}$ can be written as the ratio of two positive integers with sum at most $2 g-2$.

Example 11. The graph $\Gamma_{6}$ of Example 7 with edge lengths $m_{1}=m_{2}=\cdots=m_{6}=1$ and $\ell_{1}=\ell_{2}=\cdots=\ell_{6}=10$ is generic since $\ell_{i} / m_{i}=10 / 1$ and $10+1>2 \cdot g-2=2 \cdot 6-2=10$.

\subsection{The algorithm of Cools et al., and the bijection $\phi$}

We proceed with describing the algorithm of Cools et al. Its input, as indicated in the previous subsection, are sequences $\left(d_{0} ; x_{1}, \ldots, x_{g}\right)$ with $d_{0} \in \mathbb{Z}$ and $x_{i} \in \mathbb{R}$ that encode $v_{0}$-reduced divisors according to the scheme of Definition 8. Next, we define the objects which will constitute the algorithm's output, and afterward we specify the algorithm itself.

Definition 12. Fix a positive integer $r$. Define the Weyl chamber $\mathcal{C} \subset \mathbb{Z}^{r}$ to consist of those points $p$ for which $p(1)>p(2)>p(3)>\cdots>p(r)>0$. Then an $r$-dimensional lingering lattice path is a sequence of points $p_{0}, p_{1}, \ldots, p_{g}$ in the Weyl chamber such that:

1. $p_{0}=\left(d_{0}, d_{0}-1, d_{0}-2, \ldots, d_{0}-(r-1)\right)$ for some positive integer $d_{0}$;

2. For any $1 \leqslant i \leqslant g$, we have that each step $p_{i}-p_{i-1}$ is either one of the standard basis vectors $e_{i}$ for $\mathbb{Z}^{r}$, the negative diagonal $(-1,-1, \ldots,-1)$, or zero;

We denote the set of $r$-dimensional lingering lattice paths by $\operatorname{LLP}_{r}$, and the set of all lingering lattice paths by LLP. Note that, for the sake of brevity, our notion of lingering lattice path is more restrictive than that in [5] and captures only the objects pertinent to our algorithmic reinterpretation of their results.

An $r$-dimensional non-lingering lattice path is a lingering lattice path in which:

1. No step "lingers," in the sense that $p_{i}-p_{i-1}$ is never 0 ;

2. The total number of steps in the $(-1,-1, \ldots,-1)$ direction, which we abbreviate as the $r+1^{\text {st }}$ direction, equals the number of steps in the first direction.

3. The integer $d_{0}$ as defined in the definition of lingering lattice path is equal to $r$, so that $p_{0}=(r, r-1, \ldots, 1)$.

We denote the set of $r$-dimensional non-lingering lattice paths by $\mathrm{NLLP}_{r}$, and the set of all non-lingering lattice paths by NLLP. 
Remark 13. It is convenient to visualize the sequences $\left(p_{0}, p_{1}, \ldots, p_{g}\right) \subset \mathbb{Z}^{r}$ as a collection of $r$ piecewise-linear paths in $\mathbb{R}^{2}$ defined by requiring that for each $r \geqslant j \geqslant 1$, the points $\left(0, p_{0}(j)\right),\left(1, p_{1}(j)\right), \ldots,\left(g, p_{g}(j)\right)$ are all cusps of the $j^{\text {th }}$ path. An example of this visualization is given in Figure 3. Note that because the points of lingering lattice paths are in the Weyl chamber, they are non-intersecting with the points $\left(0, p_{0}(j)\right), \ldots,\left(g, p_{g}(j)\right)$ above $\left(\left(0, p_{0}(k)\right), \ldots,\left(g, p_{g}(k)\right)\right.$ for $j<k$.

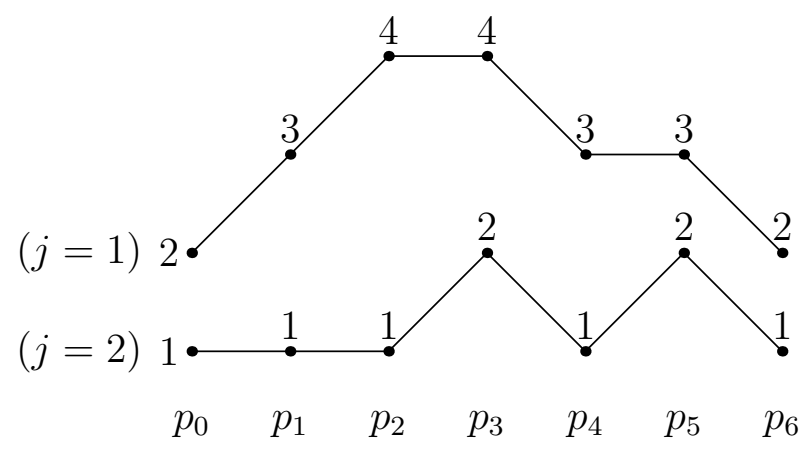

Figure 3: A 2-dimensional non-lingering lattice path

Having described the output of the algorithm, we now specify the algorithm itself and collect its combinatorial properties in the following theorem, which is a restatement of [5, Theorem 1.4] and its proof.

Algorithm 14. If $c$ is a $v_{0}$-reduced divisor given by $\left(d_{0} ; x_{1}, \ldots, x_{g}\right)$, then compute $\rho_{r}(c)=$ $\left(p_{0}, p_{1}, \ldots, p_{r}\right)$ according to the procedure:

1. $p_{0}=\left(d_{0}, d_{0}-1, \ldots, d_{0}-(r-1)\right)$;

2. $p_{i}-p_{i-1}= \begin{cases}(-1, \ldots,-1) & \text { if } x_{i}=0, \\ e_{j} & \text { if } x_{i} \equiv\left(p_{i-1}(j)+1\right) m_{i} \bmod \ell_{i}+m_{i} \\ & \text { and both } p_{i-1}, p_{i-1}+e_{j} \in \mathcal{C}, \\ 0 & \text { otherwise. }\end{cases}$

Theorem 15. Suppose that for each positive integer $r$ and generic $\Gamma_{g}$, the above algorithm, Algorithm 14, is well-defined and guaranteed to terminate, and returns a map $\rho_{r}: \operatorname{Pic}^{\leqslant 2 g-2}\left(\Gamma_{g}\right) \rightarrow \mathbb{Z}^{r}$ for generic $\Gamma_{g}$. Then, identifying each element of $\mathrm{Pic}^{\leqslant 2 g-2}\left(\Gamma_{g}\right)$ with its $v_{0}$-reduced divisor $c$, we have that $\rho_{r}(c)$ satisfies the following properties:

1. $c \in \operatorname{Pic}^{\leqslant 2 g-2}\left(\Gamma_{g}\right)$ is of rank at least $r$ if and only if $r \leqslant \max \left\{-1, d_{0}\right\}$ and $\rho_{r}(c)$ is a lingering lattice path in $\mathbb{Z}^{r}$, that is, $\rho_{r}(c)$ is in the Weyl chamber. In particular, if $\rho_{r}(c)$ is a non-lingering lattice path, then $r$ must be at most the rank of $c$ since $d_{0}=r$ so $r \leqslant \max \left\{-1, d_{0}\right\}$ clearly holds. 
2. if $\rho: \operatorname{Pic}^{\leqslant 2 g-2}\left(\Gamma_{g}\right) \rightarrow \mathrm{LLP}$ is defined by $\rho(c)=\rho_{r}(c)$ if $c$ has rank $r$, then there exists a map $\alpha: \mathrm{NLLP} \rightarrow \operatorname{Pic}\left(\Gamma_{g}\right)$ with $\rho \circ \alpha=1$, whose image consists of all rank $r$ degree $d$ elements of $\operatorname{Pic}\left(\Gamma_{g}\right)$ such that $(g-d+r)(r+1)=g$.

Example 16. The non-lingering lattice path indicated in Figure 3 is the result of applying the above algorithm to the $v_{0}$-reduced divisor of Example 7, which was given by $(2 ; 3,4,2,0,2,0)$ on the graph $\Gamma_{6}$ with edge lengths $\ell_{i}=10, m_{i}=1$.

Since $m_{i}=1$, applying the algorithm is particularly simple since $p_{i}-p_{i-1}=e_{j}$ if $x_{i} \equiv p_{i-1}(j)+1 \bmod \left(\ell_{i}+m_{i}\right)$, i.e. if $x_{i}$ is one more than $p_{i-1}(j)$ for some $j$, we increase the $j^{\text {th }}$ path. If $x_{i}$ is zero, we decrease all paths, and if $x_{i}$ is neither 0 nor one more than $p_{i-1}(j)$ for some $j$, we linger.

Thus, starting with $p_{0}=\left(\begin{array}{l}2 \\ 1\end{array}\right)$, the fact that $x_{1}=3$ and then $x_{2}=4$ give us that $p_{1}=\left(\begin{array}{l}3 \\ 1\end{array}\right)$ and then $p_{2}=\left(\begin{array}{l}4 \\ 1\end{array}\right)$. Next, the fact that $x_{3}=2$ and then $x_{4}=0$ give $p_{3}=\left(\begin{array}{l}4 \\ 2\end{array}\right)$ and then $p_{4}=\left(\begin{array}{l}3 \\ 1\end{array}\right)$. Finally, $x_{5}=2$ and $x_{6}=0$ give $p_{5}=\left(\begin{array}{l}3 \\ 2\end{array}\right)$ and $p_{6}=\left(\begin{array}{l}2 \\ 1\end{array}\right)$.

With the above theorem, Cools et al. construct their bijection $\phi$ from the map $\alpha$ and a bijection $\beta$ between rectangular standard Young tableaux and non-lingering lattice paths.

Definition 17. Let $\operatorname{SYT}\left(n^{m}\right)$ be the set of rectangular $m \times n$ standard Young tableaux, that is, $m \times n$ matrices $\left(a_{i j}\right)$ whose entries are all the integers from 1 to $m n$ such that $a_{i, j+1}, a_{i+1, j}>a_{i, j}$. Let RSYT be the set of all rectangular standard Young tableaux. See [14] for more details and background.

Proposition 18 (Proved in the proof of Theorem 1.4 of $[5])$. Let $P=\left(p_{0}, p_{1}, \ldots, p_{g}\right)$ be an $r$-dimensional non-lingering lattice path such that $g=(g-d+r)(r+1)$. We can fill $a(g-d+r) \times(r+1)$ standard Young tableau with the numbers from 1 to $g$ as follows. Starting with 1 and ending with $g$, place the number $i$ in the topmost free spot of the $j^{\text {th }}$ column if $p_{i}-p_{i-1}=e_{j}$, and in the $r+1^{\text {st }}$ column if $p_{i}-p_{i-1}=(-1, \ldots,-1)$. Furthermore, all $(g-d+r) \times(r+1)$ rectangular standard Young tableaux can be obtained in this way from one and only one non-lingering lattice path.

Remark 19. In the case $r=0$, Proposition 18 still holds. In this case, the equality $g=(g-d+r)(r+1)$ implies that $d=0$ and for each positive integer $g$, the zero divisor is indeed the unique effective divisor of degree and rank zero. This corresponds to the empty path and to the unique $g \times 1$ standard Young tableau.

Example 20. Starting with the $r=2$-dimensional non-lingering lattice path of Figure 3, we construct the standard Young tableaux as follows. From $p_{0}=\left(\begin{array}{l}2 \\ 1\end{array}\right)$ to $p_{1}=\left(\begin{array}{l}3 \\ 1\end{array}\right)$ we have an increase in the first path so we put a 1 in the first column. Going to $p_{2}=\left(\begin{array}{l}4 \\ 1\end{array}\right)$ and then to $p_{3}=\left(\begin{array}{l}4 \\ 2\end{array}\right)$ we have increases in the first and then the second path, which means we put 2 in the first column and 3 in the second. Next we have a decrease to $p_{4}=\left(\begin{array}{l}3 \\ 1\end{array}\right)$ which means we put 4 in column $r+1=3$. Continuing, we obtain the tableau:

$$
T=\begin{array}{|l|l|l|}
\hline 1 & 3 & 4 \\
\hline 2 & 5 & 6 \\
\hline
\end{array}
$$


Definition 21. Let $\beta$ : RSYT $\rightarrow$ NLLP be the inverse of the above-described bijection.

Define the injective map $\phi: \operatorname{RSYT} \rightarrow \operatorname{Pic}\left(\Gamma_{g}\right)$ by $\phi=\alpha \circ \beta: \operatorname{RSYT} \rightarrow \operatorname{Pic}\left(\Gamma_{g}\right)$. Then the map $\phi$ bijects rectangular standard Young tableaux onto rank $r$ degree $d v_{0}$-reduced divisors such that $(g-d+r)(r+1)=g$.

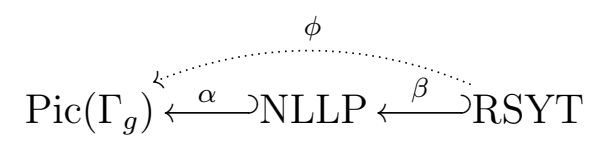

Figure 4: The map $\phi$

\section{Evacuation and Reflection}

In this section we state and prove our original result regarding how evacuation on standard Young tableaux acts on $v_{0}$-reduced divisors on the generic graphs $\Gamma_{g}$ under the bijection $\phi$ of Definition 21 discovered by Cools et al. in [5].

Definition 22. We consider an involution ev: RSYT $\rightarrow$ RSYT, called evacuation, send$\operatorname{ing} T$ in RSYT to ev $(T)=\left(b_{i j}\right)$ where $b_{i, j}=\left(m n+1-a_{m+1-i, n+1-j}\right)$. Evidently, evacuation preserves the dimensions of tableaux.

Geometrically, ev $(T)$ can be pictured as rotating the rectangular standard Young tableaux $180^{\circ}$ and flipping the entries according to the rule $i \rightarrow m n+1-i$. For more details on evacuation of tableaux, introduced by Schützenberger [13], we direct the interested reader to the wonderful survey in [14] by Richard Stanley.

Definition 23. Suppose that $\Gamma_{g}$ is as in Definition 2, i.e. the concatenation of $g$ circles $\gamma_{i}$ of circumferences $\ell_{i}+m_{i}$, along with designated vertices $\left\{v_{i}\right\}_{0 \leqslant i \leqslant g}$ such that $v_{i-1}, v_{i} \in \gamma_{i}$ and $\ell_{i}$ and $m_{i}$ are the lengths of the two arcs joining $v_{i-1}$ and $v_{i}$ in $\gamma_{i}$. Define the reflection $\Gamma_{g}^{\prime}$ of $\Gamma_{g}$ to be the same compact metric graph as $\Gamma_{g}$, but with designated vertices $v_{i}^{\prime}=v_{g+1-i}$. Note that this gives $\ell_{g+1-i}^{\prime}=\ell_{i}$ and $m_{g+1-i}^{\prime}=m_{i}$.

Identifying $\operatorname{Pic}\left(\Gamma_{g}\right)$ with the set of $v_{0}$-reduced divisors, we define the reflection $\sigma$ from $\operatorname{Pic}\left(\Gamma_{g}\right) \rightarrow \operatorname{Pic}\left(\Gamma_{g}^{\prime}\right)$ to be given by the rule that if $c$ is a $v_{0}$-reduced divisor on $\Gamma_{g}$, then $\sigma(c)$ is the $v_{g}=v_{0}^{\prime}$-reduced divisor equivalent to $c$ considered as a divisor on $\Gamma_{g}^{\prime}$.

Theorem 24. Suppose that $\Gamma_{g}$ is generic and suppose $(g-d+r)(r+1)=g$. Let $\phi=\alpha \circ \beta: \operatorname{RSYT} \rightarrow \operatorname{Pic}\left(\Gamma_{g}\right)$ be the map of Definition 21 due to Cools et al. that bijects $(g-d+r) \times(r+1)$ rectangular standard Young tableaux to rank $r$ degree $d v_{0}$-reduced divisors on $\Gamma_{g}$. Let $\phi^{\prime}=\alpha^{\prime} \circ \beta: \operatorname{RSYT} \rightarrow \operatorname{Pic}\left(\Gamma_{g}^{\prime}\right)$ be the analogous map from rectangular standard Young tableaux to $v_{0}$-reduced divisors on the reflection $\Gamma_{g}^{\prime}$. Then evacuation on 
tableaux corresponds to reflection in the sense that the following diagram commutes:

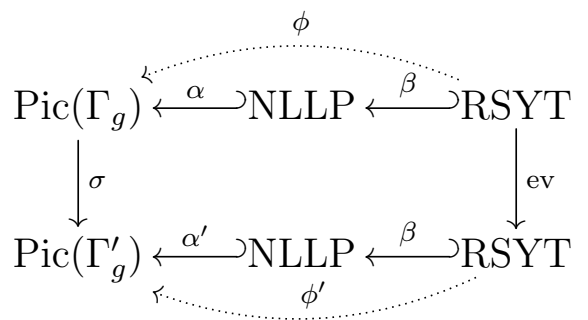

The remainder of this section is devoted to the proof this theorem. In Subsection 3.1, we introduce our useful notation for elements of $\operatorname{Pic}\left(\Gamma_{g}\right)$ in the image of $\phi$, and give formulas for $\alpha, \beta, \phi$, and $\phi^{\prime} \circ \mathrm{ev}$.

Once the notation is laid out, the heart of this proof is Proposition 36, where we characterize non-lingering lattice paths $p$ based on the sequence of differences $p_{i}-p_{i-1}$. These differences are characterized by three cases: a change in the coordinate $j$ where $j=0,0<j<r$, or $j=r$. As we will show, this characterization is symmetric in $j$ and $r-j$, so that reading the path $p^{\prime}$ obtained from reading $c$ backwards must change in the $r-j$ direction, exactly as evacuation would suggest.

In Subsection 3.2 we give a formula for $\sigma \circ \phi$, which we show is the same as the formula for $\phi^{\prime} \circ \mathrm{ev}$, thus proving Theorem 24 .

\subsection{Notation, and formulas for $\phi=\alpha \circ \beta$ and $\phi^{\prime} \circ \mathrm{ev}$}

Proposition 25 (Formula for $\alpha$ ). If $P$ is an $r$-dimensional non-lingering lattice path, then $\alpha(P)=\left(r ; x_{1}, \ldots, x_{g}\right)$ can be computed via the formula:

$$
x_{i}= \begin{cases}0 & \text { if } p_{i}-p_{i-1}=(-1,-1, \ldots,-1) \\ \left(p_{i-1}(j)+1\right) m_{i} & \text { if } p_{i}-p_{i-1}=e_{j}\end{cases}
$$

Proof. Immediate from the definition of $\alpha$ in Theorem 15 as a map that inverts $\rho$ and the definition of $\rho$.

Notation 26. If $c$ is a $v_{0}$-reduced divisor on $\Gamma_{g}$ such that $(g-d+r)(r+1)=g$, then we can describe $c$ by a sequence $\left(d_{0} ; \underline{x}_{0}, \underline{x}_{1}, \ldots, \underline{x}_{g}\right)$ where $\underline{x}_{i} m_{i}$ is the counter-clockwise distance of the single chip on $\gamma_{i}$ from $v_{i}$, if such a chip exists, and $\underline{x}_{i}=0$ if the chip does not exist. Again, this description is unique up to the modular equivalences $\underline{x}_{i} \bmod \left(\left(\ell_{i}+m_{i}\right) / m_{i}\right)$. Note that if $m_{i}=1$, we get $\underline{x}_{i} \equiv x_{i}-1 \bmod \left(\ell_{i}+m_{i}\right)$ since $x_{i}$ measures the counterclockwise distance modulo $l_{i}+1$ to the chip, starting from $v_{i-1}$, while $\underline{x}_{i}$ measures the distance starting from $v_{i}$ instead.

Using the $\underline{x}_{i}$ notation, we can thus rewrite our formula for $\alpha$ from above as:

$$
\underline{x}_{i}= \begin{cases}0 & \text { if } p_{i}-p_{i-1}=(-1,-1, \ldots,-1) \\ p_{i-1}(j) & \text { if } p_{i}-p_{i-1}=e_{j}\end{cases}
$$


Example 27. Consider once again the $v_{0}$-reduced divisor $(2 ; 3,4,2,0,2,0)$ on $\Gamma_{6}$ with $\ell_{i}=10, m_{i}=1$ from Example 7 . Since the algorithm gives a non-lingering lattice path, we know that it satisfies $(g-d+r)(r+1)=g$. In particular, it is of degree 6 and rank 2 , while the genus is 6 .

Hence in the new notation its sequence is $(2 ; \underline{2}, \underline{3}, \underline{1}, \underline{0}, \underline{1}, \underline{0})$.

Remark 28. Even though the images of $\alpha$ and $\alpha^{\prime}$ are usually different, in particular they map to divisors on $\Gamma_{g}$ and $\Gamma_{g}^{\prime}$ respectively, the images of $\phi$ and $\phi^{\prime}$ agree as tuples $\left(r ; \underline{x}_{1}, \ldots \underline{x}_{r}\right)$. Technically the tuples in the image of $\phi$ is defined modulo $\left(\ell_{i}+m_{i}\right) / m_{i}$ and the image of $\phi^{\prime}$ is defined modulo $\left(\ell_{g+1-i}+m_{g+1-i}\right) / m_{g+1-i}$, but since each $\ell_{i}+m_{i}$ is assumed to be greater than $2 g-2$, if we assume each $m_{i}=1$, then we can take the distances to satisfy $0 \leqslant \underline{x}_{i} \leqslant\left(\ell_{i}+1\right)$ so that the moduli never come into play. For other choices of $m_{i}$, the distance chosen might need to be larger than $\left(\ell_{i}+m_{i}\right) / m_{i}$, i.e. wrap around the loop $\gamma_{i}$, to agree with the values from (1). For convenience, we will assume distances are chosen to be multiples of $m_{i}$ and agreeing with the values from (1) throughout the rest of this paper.

Proposition 29 (Formula for $\beta$ ). Suppose that $T$ is a rectangular $(g-d+r) \times(r+1)$ standard Young tableau and that $\beta(T)=P=\left(p_{0}, \ldots, p_{g}\right)$. Then for any $i, p_{i}$ can be computed according to the formula:

$$
p_{i}=p_{0}+\left(\begin{array}{c}
l_{1}-l_{r+1} \\
l_{2}-l_{r+1} \\
\ldots \\
l_{r}-l_{r+1}
\end{array}\right)=\left(\begin{array}{c}
r+l_{1}-l_{r+1} \\
r-1+l_{2}-l_{r+1} \\
\ldots \\
1+l_{r}-l_{r+1}
\end{array}\right)
$$

where $l_{s}$ is the number of cells in the $s^{\text {th }}$ column of $T$ whose entries are at most $i$.

Proof. In the bijection of Cools et al. (see Proposition 18) between the non-lingering lattice paths and the standard Young tableaux, a number $k \leqslant i$ is placed in column $j<r+1$ when $p_{k}-p_{k-1}=e_{j}$, i.e., when there has been an increase in the $j^{\text {th }}$ direction. The number $l_{j}$ hence counts the number of increases that have occurred in the $j^{\text {th }}$ direction by the $i^{\text {th }}$ step.

On the other hand, a number $k \leqslant i$ is placed in column $r+1$ when $p_{k}-p_{k-1}=$ $(-1,-1, \ldots,-1)$, i.e., when there has been a decrease along all directions. Hence, $l_{r+1}$ counts the number of decreases that have occurred by the $i^{\text {th }}$ step. Knowing that we start with $p_{0}=(r, r-1, \ldots, 1)$, it follows that $p_{i}(j)=p_{0}(j)+l_{j}-l_{r+1}$ and hence the proposition follows.

Notation 30. If $T$ is a rectangular $m \times n$ standard Young tableau and $1 \leqslant i \leqslant m n$, then we let

(i) $l_{r}(i, T)$ denote the index of the row of $T$ (from top to bottom) containing $i$,

(ii) $l_{c}(i, T)$ denote the index of the column (from left to right) containing $i$, 
(iii) $L_{\text {first }}(i, T)$ denote the number of cells in the first column whose entries are strictly less than $i$, and

(iv) $L_{\text {last }}(i, T)$ denote the number of cells in the last column whose entries are strictly less than $i$.

Putting the formulas for $\alpha$ and $\beta$ together, we obtain a formula for $\phi=\alpha \circ \beta$.

Proposition 31 (Formula for $\phi)$. Suppose that $T \in \operatorname{SYT}\left((r+1)^{g-d+r}\right)$, and that $\phi(T)=$ $\alpha \circ \beta(T)=c$ is described by $\left(r ; \underline{x}_{1}, \ldots, \underline{x}_{g}\right)$.

Then the $\underline{x}_{i}$ 's can be computed according to the formula:

$$
\underline{x}_{i}=r+l_{r}(i, T)-l_{c}(i, T)-L_{l a s t}(i, T)
$$

using the above notation.

Proof. Let $\beta(T)=P=\left(p_{0}, \ldots, p_{g}\right)$ so that $\alpha(P)=c$. If the number $i$ is in the $l=$ $l_{c}(i, T)^{\text {th }}$ column of $T$ for $1 \leqslant l<r+1$, then $p_{i}-p_{i-1}=e_{l}$ and hence formula (1) for $\alpha$ gives us $\underline{x}_{i}=p_{i-1}(l)$, while formula (2) for $\beta$ gives us

$$
\left.p_{i-1}(l)=(r+1-l)+\left(l_{r}(i, T)-1\right)-L_{\text {last }}(i, T)\right),
$$

yielding formula (3). Note that since $l_{r}(i, T)$ is the index of the row containing $i$, it follows that $l_{r}(i, T)-1$ is the number of entries in column $l$ that are at most $i-1$.

Otherwise, if the number $i$ is in the $r+1^{\text {st }}$ column of $T$, then $p_{i}-p_{i-1}=(-1, \ldots,-1)$. We obtain $\underline{x}_{i}=0$ in this case, and since $l_{c}(i, T)=r+1$ and $l_{r}(i, T)=L_{\text {last }}(i, T)+1$, the proposed formula for $\phi$ still holds.

Example 32. Consider the tableau of Example 20

$$
T=\begin{array}{|l|l|l|}
\hline 1 & 3 & 4 \\
\hline 2 & 5 & 6 \\
\hline
\end{array}
$$

Using the formula for $\phi$ from Proposition 31, we compute $\phi(T)=(2 ; \underline{2}, \underline{3}, \underline{1}, \underline{0}, \underline{1}, \underline{0})$ agreeing with Example 27. To compute $\underline{x}_{5}$, for example, we see that $i=5$ is in row $2=l_{r}(5, T)$ and column $2=l_{c}(5, T)$. Also, the number of cells in the last columns that are strictly less than $i=5$ is $L_{\text {last }}(5, T)=1$. It follows that $\underline{x}_{5}=r+l_{r}(5, T)-l_{c}(5, T)-$ $L_{\text {last }}(5, T)=2+2-2-1=1$.

Next, we obtain the formula for $\phi^{\prime} \circ \mathrm{ev}$.

Proposition 33 (Formula for $\left.\phi^{\prime} \circ \mathrm{ev}\right)$. Suppose that $T$ is a $(g-d+r) \times(r+1)$ rectangular standard Young tableaux and that $\phi^{\prime} \circ \mathrm{ev}(T)=\alpha^{\prime} \circ \beta \circ \mathrm{ev}(T)=c^{\prime}$ is described by $\left(r ; \underline{x}_{1}^{\prime}, \ldots, \underline{x}_{g}^{\prime}\right)$.

Then the $\underline{x}_{i}^{\prime}$ 's can be computed according to the formula:

$$
\underline{x}_{g+1-i}^{\prime}=j-1+l_{1}-l_{j}
$$

where $j=l_{c}(i, T)$ is the index of the column containing $i$ and $l_{s}$ is the number of cells in the $s^{\text {th }}$ column of $T$ whose entries are at most $i$. 
Proof. Evacuation takes column $j=l_{c}(i, T)$ of $T$ to column $r+2-j$. Hence, if $l_{j}$ is the number of cells in column $j$ of $T$ whose entries are at most $i$, then $l_{j}$ is also the number of cells in column $r+2-j$ of ev $(T)$ whose entries are at least $g+1-i$ (since evacuation also flips the values of the entries).

If we let $\beta(\operatorname{ev}(T))=\left(p_{0}^{\prime}, p_{1}^{\prime}, \ldots, p_{g}^{\prime}\right)$, then the $l_{s}$ acquire the following significance. For $j \neq 1, l_{j}$ counts the number of increases in the $r+2-j^{\text {th }}$ direction from $p_{g-i}^{\prime}$ to $p_{g}^{\prime}=(r, r-1, \ldots, 1)$. Similarly, $l_{1}$ counts the number of decreases along all directions from $p_{g-i}^{\prime}$ to $p_{g}^{\prime}=(r, r-1, \ldots, 1)$.

Given that the $l_{j}$ are counting steps in the $r+2-j^{\text {th }}$ direction from $p_{g-i}^{\prime}$ to $p_{g}^{\prime}$, we obtain the following analogue of Proposition 29:

$$
p_{g-i}^{\prime}=p_{g}^{\prime}-\left(\begin{array}{c}
l_{r-1}-l_{1} \\
l_{r}-l_{1} \\
\ldots \\
l_{2}-l_{1}
\end{array}\right)=\left(\begin{array}{c}
r+l_{1}-l_{r+1} \\
r-1+l_{1}-l_{r} \\
\ldots \\
1+l_{2}-l_{1}
\end{array}\right) .
$$

Next we obtain the analogue of Proposition 31 using exactly the same argument. Suppose that $i$ is in the $j^{\text {th }}$ column of $T$. Then we have that $g+1-i$ is in the $r+2-j^{\text {th }}$ column of ev(T). If $j \geqslant 2$, then $p_{g+1-i}^{\prime}-p_{g-i}^{\prime}=e_{r+2-j}$ and hence $x_{g+1-i}^{\prime}=p_{g-i}^{\prime}(r+2-j)=$ $j-1+l_{1}-l_{j}$ where $l_{s}$ is the number of cells in the $s^{\text {th }}$ column of $T$ whose entries are at most $i$.

Otherwise, if $j=1$, we have that $g+1-i$ is in the $r+1^{\text {st }}$ column of ev(T), which means that $p_{g+1-i}^{\prime}-p_{g-i}^{\prime}=(-1, \ldots,-1)$ and $x_{g+1-i}^{\prime}=0$. Since $0=1-1+l_{1}-l_{1}$, the formula holds in both cases.

Example 34. Consider the tableau of Example 20 and its associated non-lingering lattice path $P^{\prime}=\beta(\operatorname{ev}(T))$ shown in Figure 5 .

$$
T=\begin{array}{|l|l|l|}
\hline 1 & 3 & 4 \\
\hline 2 & 5 & 6 \\
\hline
\end{array} \quad \quad \operatorname{ev}(T)=\begin{array}{|l|l|l|}
\hline 1 & 2 & 5 \\
\hline 3 & 4 & 6 \\
\hline
\end{array}
$$

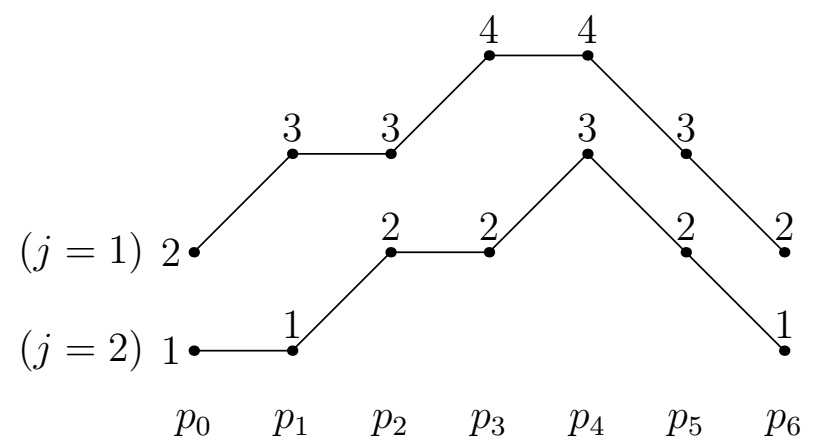

Figure 5: The 2-dimensional non-lingering lattice path $P^{\prime}$ associated to ev $(T)$.

This example will demonstrate the shortcut formula for $\phi^{\prime} \circ \mathrm{ev}$ of Proposition 33. To compute $\underline{x}_{3}^{\prime}$, for example, we see that $g+1-i=3$ implies $i=7-3=4$, and that 
4 is in column $3=j$. The number $l_{3}$ of cells in the third column that are at most $i=4$ is 1 , and the number of cells $l_{1}$ in the first column that are at most $i=4$ is 2 . It follows that $\underline{x}_{3}=j-1+l_{1}-l_{j}=3-1+2-1=3$. Doing this for each $\underline{x}_{i}$, we obtain the sequence $c^{\prime}=\alpha^{\prime}\left(P^{\prime}\right)=(2 ; \underline{2}, \underline{1}, \underline{3}, \underline{2}, \underline{0}, \underline{0})$. In Example 37, we will show that this sequence corresponds to the reflection $\sigma\left(c^{\prime}\right)$ of the $v_{0}$-reduced divisor $(2 ; \underline{2}, \underline{3}, \underline{1}, \underline{0}, \underline{1}, \underline{0})$ from Example 27.

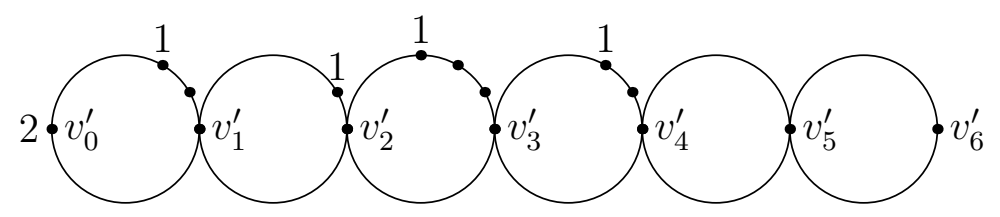

Figure 6: The proposed reflection of the $v_{0}$-reduced divisor from Example 7

\subsection{Formula for $\sigma \circ \alpha$ and proof of Theorem 24}

We compute a formula for $\sigma \circ \alpha$ using the following lemma.

Lemma 35. Let $c$ be a $v_{0}$-reduced divisor of non-negative degree at most $2 g-2$, and for $i \geqslant 0$ let $c_{i}$ be the $v_{i}$-reduced divisor that is equivalent to $c$. Then if $\rho(c)=P=$ $\left(p_{0}, p_{1}, \ldots, p_{g}\right)$, we have that $c_{i}\left(v_{i}\right)=p_{i}(1)$.

Proof. Trivially, we have that $p_{0}(1)=d_{0}=c_{0}\left(v_{0}\right)$.

Next, suppose inductively that $p_{i-1}(1)=k=c_{i-1}\left(v_{i-1}\right)$. By Lemma 9 we know that $c_{i}\left(v_{i}\right)-c_{i-1}\left(v_{i-1}\right)=-1,0$, or 1 depending on whether $x_{i}=0, x_{i} \equiv(k+1) m_{i} \bmod \left(\ell_{i}+m_{i}\right)$, or $x_{i} \not \equiv(k+1) m_{i} \bmod \left(\ell_{i}+m_{i}\right)$. But since $p_{i-1}(1)=k$, these are precisely the conditions for $p_{i}-p_{i-1}=(-1,-1, \ldots,-1), 0$, or $e_{1}$, and hence for $p_{i}(1)-p_{i-1}(1)=-1,0$, or 1 .

Proposition 36 (Formula for $\sigma \circ \alpha)$. Suppose that $c \in \operatorname{Pic}\left(\Gamma_{g}\right)$ is such that $c=\alpha(P)$ for some non-lingering lattice path $P=\left(p_{0}, p_{1}, \ldots, p_{g}\right)$, i.e. that $c$ is of rank $r$ and degree $d$ such that $(g-d+r)(r+1)=g$, and further that $c$ is described by a sequence $\left(r ; \underline{x}_{1}, \ldots, \underline{x}_{g}\right)$.

Then $\sigma(c)$ is also of rank $r$ and degree $d$ such that $(g-d+r)(r+1)=g$, and hence can be described by a sequence $\left(r ; \underline{x}_{1}^{\prime}, \ldots, \underline{x}_{g}^{\prime}\right)$. The $\underline{x}_{i}^{\prime}$ 's can be computed from the $\underline{x}_{i}$ 's using the formula:

$$
\underline{x}_{g+1-i}^{\prime}=\max \left\{p_{i-1}(1)-\underline{x}_{i}-1,0\right\} .
$$

Proof. Note that under the reflection that takes $\Gamma_{g}$ to $\Gamma_{g}^{\prime}$, the counter-clockwise distances from $v_{i}$ in the loop $\gamma_{i}$ are sent to clockwise distance from $v_{1+g-i}^{\prime}=v_{i}$ in the loop $\gamma_{g+1-i}^{\prime}=$ $\gamma_{i}$. Hence, the $\underline{x}_{i}^{\prime}$ s in the sequence $\left(r ; \underline{x}_{1}^{\prime}, \ldots, \underline{x}_{g}^{\prime}\right)$ which describes $\sigma(c)$, the $v_{g}=v_{0}^{\prime}$-reduced divisor equivalent to $c$, can be interpreted as both the counter-clockwise distance from $v_{i}^{\prime}$ in the loop $\gamma_{i}^{\prime}$, and as the clockwise distances from $v_{1+g-i}$ of the single chip on the loop $\gamma_{1+g-i}$. 
These clockwise distances, however, are determined by successively computing the equivalent $v_{1}$-reduced divisor, then the equivalent $v_{2}$-reduced divisor and so on until the equivalent $v_{g}$-reduced divisor. Lemma 9 applies and gives us the following.

Suppose that $k$ is the number of chips on $v_{i-1}$ of the $v_{i-1}$-reduced divisor $c_{i-1}$ that is equivalent to $c$. Then combining Lemma 9 with our notation for $\underline{x}_{i}$, we obtain:

1. if $\underline{x}_{i}$ is 0 , i.e. if there is no chip on $\gamma_{i} \backslash\left\{v_{i-1}\right\}$ in $c_{i-1}$, then there will be one chip on $\gamma_{i} \backslash\left\{v_{i}\right\}$ that is a clockwise distance $(k-1) m_{i}$ away from $v_{i-1}$ in $c_{i}$;

2. if $\underline{x}_{i} m_{i} \not \equiv k m_{i} \bmod \left(l_{i}+m_{i}\right)$, then there is one chip on $\gamma_{i} \backslash\left\{v_{i}\right\}$ in $c_{i-1}$ that is a clockwise distance $k m_{i}-\left(\underline{x}_{i}+1\right) m_{i}$ away from $v_{i-1}$ in $c_{i}$;

3. if $\underline{x}_{i} m_{i} \equiv k m_{i} \bmod \left(l_{i}+m_{i}\right)$, then there are no chips left on $\gamma_{i} \backslash\left\{v_{i}\right\}$ in $c_{i}$.

Now, $k$ is of course $p_{i-1}(1)$ by the previous lemma. Hence, the formula $\underline{x}_{g+1-i}^{\prime}=$ $\max \left\{p_{i-1}(1)-\underline{x}_{i}-1,0\right\}$ holds for each of the three cases above.

Example 37. Consider the $v_{0}$-reduced divisor $c=\left(r ; \underline{x}_{1}, \ldots, \underline{x}_{g}\right)=(2 ; \underline{2}, \underline{3}, \underline{1}, \underline{0}, \underline{1}, \underline{0})$ on the graph $\Gamma_{6}$ from Example 27. Its associated non-lingering lattice path is the one from Figure 3 with top path $\left(p_{0}(1), p_{1}(1), \ldots, p_{g}(1)\right)=(2,3,4,4,3,3,2)$.

Figure 7 illustrates the process described in the proof of Proposition 36 of successively computing the $v_{i}$-reduced divisors $c_{i}$ equivalent to $c$. Looking at the top path $(2,3,4,4,3,3,2)$, the figure also illustrates the claim of Lemmas 9 and 35.

To illustrate Proposition 36, note that if we subtract the sequence of $\underline{x}_{i}$ 's in $c=$ $(2 ; \underline{2}, \underline{3}, \underline{1}, \underline{0}, \underline{1}, \underline{0})$ from the sequence of $p_{i-1}(1)$ 's for $1 \leqslant i \leqslant g$, which is $(2,3,4,4,3,3)$, we obtain $(0,0,3,4,2,3)$. Subtracting a further 1 from everything and reversing, we get $(2,1,3,2,-1,-1)$. Taking the maximum with 0 and putting the rank $r=2$ in front, we obtain the $v_{0}^{\prime}$-reduced divisor on $\Gamma_{g}^{\prime}$ given by $\left(r ; \underline{x}_{1}^{\prime}, \ldots, \underline{x}_{g}^{\prime}\right)=(2 ; \underline{2}, \underline{1}, \underline{3}, \underline{2}, \underline{0}, \underline{0})$, which is what Figure 7 also produces.

In accordance with our theorem, this is exactly the same $v_{0}^{\prime}$-reduced divisor as the one obtained from evacuating the tableau in Example 34.

Proof of Theorem 24. We need to show that $\phi^{\prime} \circ \mathrm{ev}=\sigma \circ \phi$, and we have so far formulas for $\phi^{\prime} \circ$ ev, $\sigma \circ \beta$, and $\alpha$ where $\phi=\beta \circ \alpha$.

We proceed to obtain a formula for $\sigma \circ \phi$, which we then reduce to the formula for $\phi^{\prime} \circ \mathrm{ev}$.

Suppose that $T$ is a rectangular standard Young tableaux. Then let $\beta(T)=P=$ $\left(p_{0}, \ldots, p_{g}\right), \phi(T)=\alpha \circ \beta(T)=\alpha(P)=\left(r ; \underline{x}_{1}, \ldots, \underline{x}_{g}\right)$ and $\sigma \circ \alpha(P)=\left(r ; \underline{x}_{1}^{\prime}, \ldots, \underline{x}_{g}^{\prime}\right)$. We have established that:

1. $\underline{x}_{g+1-i}^{\prime}=\max \left\{p_{i-1}(1)-\underline{x}_{i}-1,0\right\}$ by the formula (5) for $\sigma \circ \alpha$.

2. $p_{i-1}(1)=r+L_{\text {first }}(i, T)-L_{\text {last }}(i, T)$ by the formula $(2)$ for $\beta$ (noting that $i$ is not necesarrily in column 1 ).

3. $\underline{x}_{i}=r+l_{r}(i, T)-l_{c}(i, T)-L_{\text {last }}(i, T)$ by the formula (3) for $\phi$. 

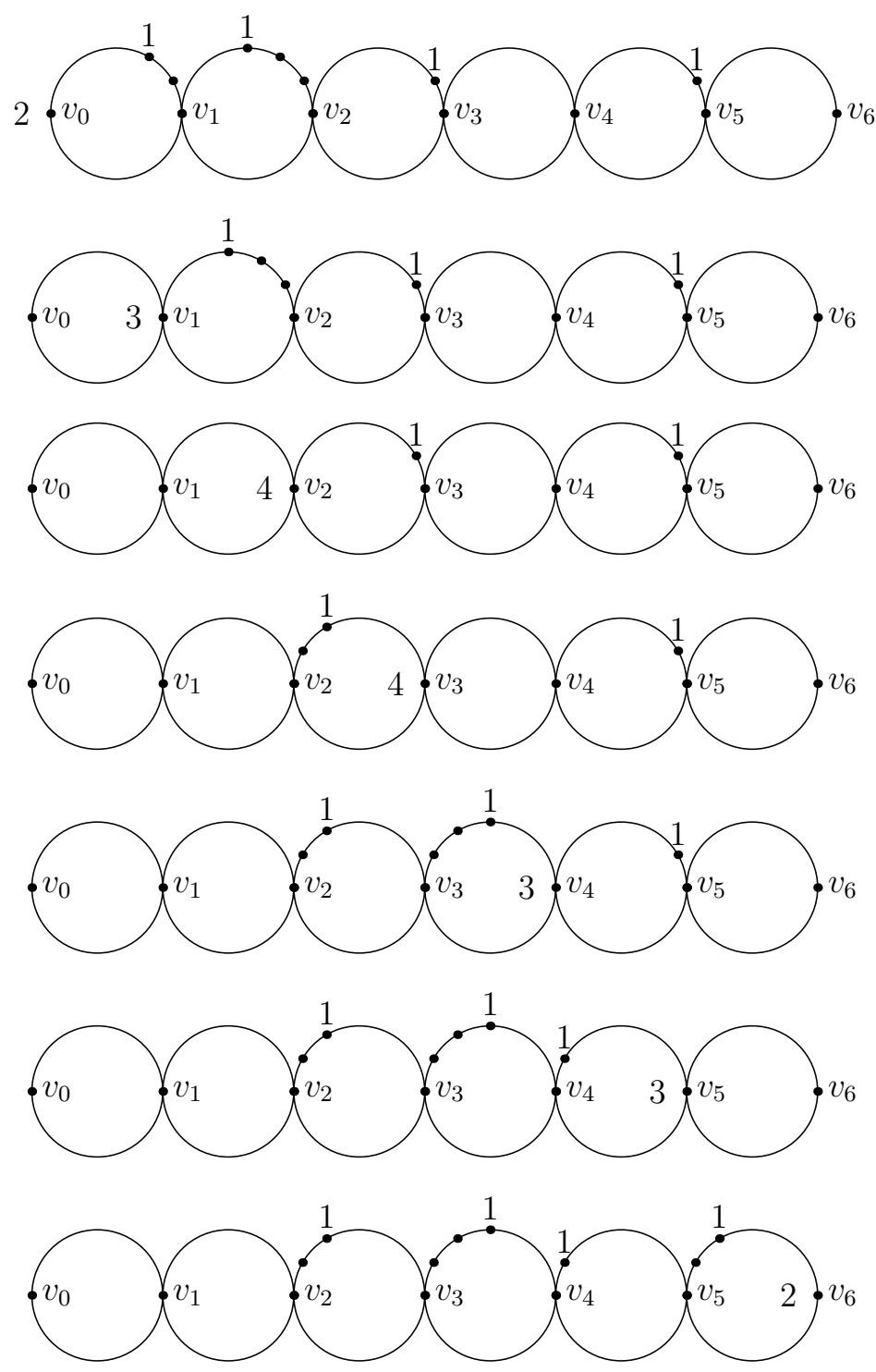

We reflect by setting $v_{i}=v_{g+1-i}^{\prime}$, and obtain the configuration illustrated in Figure 6:
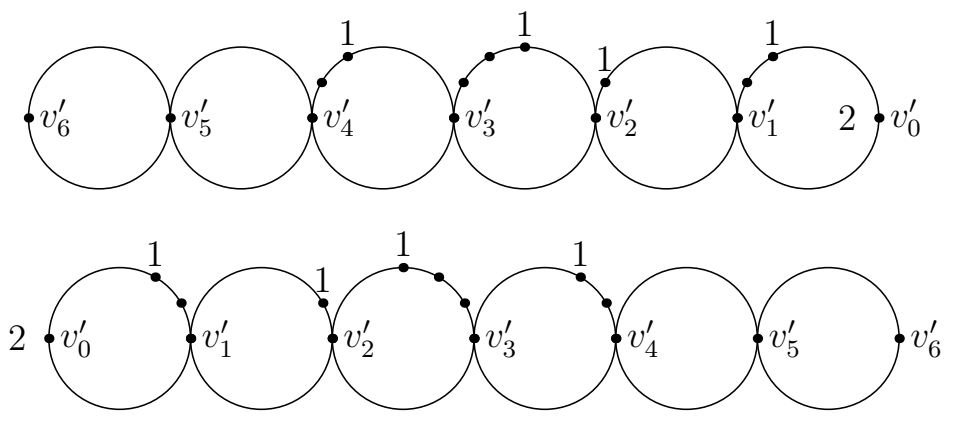

Figure 7: Successively computing $v_{i}$-reduced divisors 
where the notation $L_{\text {first }}(i, T), L_{\text {last }}(i, T), l_{r}(i, T)$, and $l_{c}(i, T)$ is as above. Thus

$$
\begin{aligned}
\underline{x}_{g+1-i}^{\prime}= & \max \left\{\left(r+L_{\text {first }}(i, T)-L_{\text {last }}(i, T)\right)\right. \\
& \left.-\left(r+l_{r}(i, T)-l_{c}(i, T)-L_{\text {last }}(i, T)\right)-1,0\right\} \\
= & \max \left\{L_{\text {first }}(i, T)-l_{r}(i, T)+l_{c}(i, T)-1,0\right\} .
\end{aligned}
$$

Note that if $l_{c}(i, T)=1$, then the number of cells in column 1 whose entries are strictly less than $i$ is one less than the index of the row containing $i$. We thus obtain $\underline{x}_{g+1-i}^{\prime}=$ $\max \left\{L_{\text {first }}(i, T)-l_{r}(i, T), 0\right\}=\max \{-1,0\}=0$. Comparing this with formula (4), we see $l_{c}(i, T)=1$ and indeed $0=l_{c}(i, T)-1+l_{1}-l_{1}$.

On the other hand, if $i$ is in column $j \neq 1$, then $l_{1}=L_{\text {first }}(i, T)$ and $l_{j}=l_{r}(i, T)$. Since $j-1+l_{1}-l_{j}$ is certainly non-negative as it is the formula (4) for $\phi^{\prime} \circ \mathrm{ev}$, we have $\underline{x}_{g+1-i}^{\prime}=j-1+l_{1}-l_{j}$ as desired in this case as well.

Hence, we have proven that the formulas for $\sigma \circ \phi$ and $\phi^{\prime} \circ$ ev agree.

\section{Conjugation of Tableaux and Riemann-Roch Du- ality}

We now prove a conjecture based on discussions with Sam Payne [11].

Definition 38. We consider an involution $t:$ RSYT $\rightarrow$ RSYT, called conjugation (also known as transposition), sending $T=\left(a_{i j}\right)$ in $R S Y T$ to $T^{t}=\left(a_{j i}\right)$.

Unlike evacuation, the dimensions of a tableau $T$ are not fixed under conjugation. In particular, an $m \times n$ standard Young tableau is sent to an $n \times m$ standard Young tableau. Consequently, the corresponding divisors $\phi(T)$ and $\phi\left(T^{t}\right)$, have different ranks and degrees. Nevertheless, there is a natural duality induced by this involution on rectangular standard Young tableaux.

Theorem 39. Suppose that $g=(g-d+r)(r+1)$ and that $T$ is a rectangular $(g-d+$ $r) \times(r+1)$ standard Young tableau. Let $\phi(T)=\left(r ; \underline{x}_{1}, \ldots, \underline{x}_{g}\right)$, a rank $r$ and degree $d$ divisor $c$ in $\operatorname{Pic}\left(\Gamma_{g}\right)$.

Then $\phi\left(T^{t}\right)$ is linearly equivalent to the rank $g-d+r-1$ and degree $2 g-2-d$ divisor $K-c$ in Pic $\left(\Gamma_{g}\right)$. Here $K$ is the canonical divisor on $\Gamma_{g}$ that appears in the Tropical Riemann-Roch Theorem (Theorem 1).

To prove Theorem 39, it suffices to prove the following two propositions.

Proposition 40. Assume the hypotheses of Theorem 39, including the equality $\phi(T)=$ $\left(r ; \underline{x}_{1}, \ldots, \underline{x}_{g}\right)$. Define tuples $\left(\underline{z}_{0}, \underline{z}_{1}, \ldots, \underline{z}_{g-1}\right)$ and $\left(\underline{y}_{1}, \underline{y}_{2}, \ldots, \underline{y}_{g}\right)$ as follows. For $0 \leqslant i \leqslant$ $g-1$, let $z_{i}=\#\{j>i$ in the last row or last column of $T\}+1$. Then, for $1 \leqslant i \leqslant g$, define $\underline{y}_{i}$ as

$$
\underline{y}_{i}=z_{i-1}-\underline{x}_{i}-2 \text {. }
$$

Then we obtain $\phi\left(T^{t}\right)=\left(s ; \underline{y}_{1}, \ldots, \underline{y}_{g}\right)$ where $s=g-d+r-1$. 
Proposition 41. Let $K$ be the canonical divisor on $\Gamma_{g}$, let $c$ be the $v_{0}$-reduced divisor in Pic $\left(\Gamma_{g}\right)$ represented by $\left(r ; \underline{x}_{1}, \ldots, \underline{x}_{g}\right)$, and let $\left(s ; \underline{y}_{1}, \ldots, \underline{y}_{g}\right)$ be as defined in Proposition 40. Then the $v_{0}$-reduced divisor equivalent to $K-c$ in Pic $\left(\Gamma_{g}\right)$ is represented by $\left(s ; \underline{y}_{1}, \ldots, \underline{y}_{g}\right)$.

Proof of Proposition 40. Let $T^{t}$ denote the conjugate of $T$. Following the logic of Proposition 31, the first value of tuple $\phi\left(T^{t}\right)$ is one less than the number of columns of $T^{t}$. Using the fact that $T^{t} \in S Y T\left((g-d+r)^{(r+1)}\right)$, we obtain that this value is $s=g-d+r-1$ as desired.

It next suffices to show the equality $\underline{x}_{i}+\underline{y}_{i}=z_{i-1}-2$ for all $1 \leqslant i \leqslant g$. Using formula (3) for the $\underline{x}_{i}$ 's and switching the roles of "rows" and "columns", or $T$ and $T^{t}$, to obtain a formula for the $\underline{y}_{i}^{\text {'s yields }}$

$$
\begin{aligned}
\underline{x}_{i}+\underline{y}_{i}= & \left(r+l_{r}(i, T)-l_{c}(i, T)-L_{\text {last }}(i, T)\right) \\
& \quad+\left(s+l_{c}(i, T)-l_{r}(i, T)-L_{\text {last }}\left(i, T^{t}\right)\right) \\
= & r+s-L_{\text {last }}(i, T)-L_{\text {last }}\left(i, T^{t}\right),
\end{aligned}
$$

where $L_{\text {last }}(i, T)$ (resp. $L_{\text {last }}\left(i, T^{t}\right)$ ) equals the number of cells in the last column of $T$ (resp. $T^{t}$ ) whose entries are strictly less than $i$. It follows that

$$
\begin{aligned}
& (s+1)-L_{\text {last }}(i, T)=\#(\text { cells }>(i-1) \text { in the last column of } \mathrm{T}), \text { and } \\
& (r+1)-L_{\text {last }}\left(i, T^{t}\right)=\#(\text { cells }>(i-1) \text { in the last row of } \mathrm{T}) .
\end{aligned}
$$

Adding these two together, and subtracting 2, we conclude that

$$
\underline{x}_{i}+\underline{y}_{i}=r+s-L_{\text {last }}(i, T)-L_{\text {last }}\left(i, T^{t}\right)=z_{i-1}-2
$$

as desired (keeping in mind that $z_{i-1}$ double-counts the unique cell in the last row and column).

Before proving Proposition 41, we need one Lemma.

Lemma 42. Let $T$ be an $(r+1) \times(s+1)$ rectangular standard Young tableau, and suppose that $\phi(T)=\left(r ; \underline{x}_{1}, \underline{x}_{2}, \ldots, \underline{x}_{g}\right)$. Define $z_{i}$ as in Proposition 40. Then for $1 \leqslant i \leqslant g-1$, we have $\underline{x}_{i}=0$ if the entry $i$ is in the last column of $T$ and $\underline{x}_{i}=z_{i}-1$ if the entry $i$ is in the last row of $T$. If the entry $i$ is in neither the last row nor the last column, then $0<\underline{x}_{i}<z_{i}-2$. Note in particular that $\underline{x}_{i}=z_{i}-2$ is not possible.

Proof. The first statement was already shown in the proof of Proposition 31. To prove the second statement, assume that $i$ in the last row of $T$. From formula (3), we obtain $\underline{x}_{i}=r+(s+1)-l_{c}(i, T)-L_{\text {last }}(i, T)$ in this case. Noting that $r+s+1$ equals the number of cells in the last row or last column of $T$, we see that $\underline{x}_{i}$ counts the number of cells in the last row or last column greater than $i$. However, this is exactly the definition of $z_{i}-1$ and hence the second statement is proven. 
In the event that the entry $i$ is in neither the last row nor the last column, then $\left.\underline{x}_{i}=(r+s+1)-l_{c}(i, T)-L_{\text {last }}(i, T)\right)-\alpha<z_{i}-1-\alpha$ where $\alpha=s+1-l_{r}(i, T) \geqslant 1$. Note that we have an inequality on the RHS instead of an equality this time because if $i$ is in column $l_{c}(i, T)$ then the bottom row of that column is greater than $i$ as opposed to merely equal to $i$. We could additionally have entries in the bottom row to the left of column $l_{c}(i, T)$ that are greater than $i$, thus we have the desired inequality.

Proof of Proposition 41. We first note that for graph $\Gamma_{g}$, the canonical divisor $K$ is simply given as $2 v_{1}+2 v_{2}+\cdots+2 v_{g-1}$. Hence, the degree of $K-c$ is $d^{t}=2(g-1)-d$ and the rank is $r^{t}=g-d+r-1=s$ by the Tropical Riemann-Roch Theorem. In particular, we still have the equality $\left(g-d^{t}+r^{t}\right)\left(r^{t}+1\right)=g$ since $\left(g-d^{t}+r^{t}\right)\left(r^{t}+1\right)=$ $(g-(2 g-2-d)+(g-d+r-1))(g-d+r)=(r+1)(g-d+r)$.

Secondly, we take $K-c$, where $c$ is represented by $\left(r ; \underline{x}_{1}, \underline{x}_{2}, \ldots, \underline{x}_{g}\right)$, and compute its $v_{0}$-reduction by successively firing the subgraphs $\gamma_{g}, \gamma_{g-1} \cup \gamma_{g}$, etc. enough times from right to left. We define a sequence $\left(Z_{g-1}, Z_{g-2}, \ldots, Z_{0}\right)$ by letting $Z_{g-i}$ denote the number of chips on vertex $v_{g-i}$ after the subgraph $\gamma_{g-i+2} \cup \gamma_{g-i+3} \cup \cdots \cup \gamma_{g}$ has been fired enough times. In particular, $Z_{g-1}=2$ since in the divisor $K-c$, the vertex $v_{g-1}$ (before any firing) has 2 chips on it. We also obtain $Z_{g-2}=3$ since $\gamma_{g}$ contains 2 chips on $v_{g-1}$, and nowhere else, so after $\gamma_{g}$ is fired, there are 3 chips on vertex $v_{g-2}$.

We also define the sequence $\left(Y_{g}, Y_{g-1}, \ldots, Y_{1}\right)$ by letting $Y_{g-i}$ denote the counterclockwise distance from $v_{g-i}$ of the unique chip on the loop $\gamma_{g-i} \backslash\left\{v_{g-i-1}, v_{g-i}\right\}$ after $\gamma_{g-i+1} \cup \gamma_{g-i+2} \cup \cdots \cup \gamma_{g}$ has been fired, with the convention that $Y_{g-i}=0$ if no such chip exists. For example, the entry $g$ must be in the unique cell in the last row and last column, thus $\underline{x}_{g}=r+(s+1)-(r+1)-s=0$ by formula (3). Consequently, the divisor $K-c$ has no chips on $\gamma_{g} \backslash\left\{v_{g-1}, v_{g}\right\}$, and we obtain $Y_{g}=0$ (no firings have yet taken place).

We next observe that if $\underline{x}_{g-1}=1$, we fire $\gamma_{g}$ and obtain $Y_{g-1}=0$ while if $\underline{x}_{g-1}=0$, we instead obtain $Y_{g-1}=1$ after firing $\gamma_{g}$. Note that formula (3) yields $\underline{x}_{g-1}=r+s-(r+$ $1)-(s-1)=0\left(\operatorname{resp} . \underline{x}_{g-1}=r+(s+1)-r-s=1\right)$ if $(g-1)$ is above (resp. to the left of) the cell containing $g$. Since entry $(g-1)$ must be in one of the two cells next to entry $g$, no other values for $\underline{x}_{g-1}$ are possible.

Having fired subgraphs $\gamma_{g-i+2} \cup \gamma_{g-i+3} \cup \cdots \cup \gamma_{g}$ to clear chips from vertices successively from $v_{g-i+1}$ to $v_{g}$ and ensure that there are no negative chips to the right of $v_{g-i}$, we next focus on the loop $\gamma_{g-i}$. Inductively, it contains $Z_{g-i}$ chips at $v_{g-i}, 2$ chips at $v_{g-i-1}$ and -1 chips a counter-clockwise distance of $\underline{x}_{g-i}$ from $v_{g-i}$ (unless $\underline{x}_{g-i}=0$ in which case the only chips are at $v_{g-i}$ and $\left.v_{g-i-1}\right)$ at this point. Firing the subgraph $\gamma_{g-i+1} \cup \gamma_{g-i+2} \cup \cdots \cup \gamma_{g}$, for $1 \leqslant i \leqslant g-1$, we have three cases:

i) if $\underline{x}_{g-i}=0$, then $Z_{g-i-1}=Z_{g-i}+1$ and we have one chip left a counter-clockwise distance $Y_{g-i}=Z_{g-i}-1$ from $v_{g-i}$.

ii) if $\underline{x}_{g-i}=Z_{g-i}-1$, then we can move all of the chips to the left so we obtain $Z_{g-i-1}=Z_{g-i}+1$ and $Y_{g-i}=0$.

iii) if $0<\underline{x}_{g-i}<Z_{g-i}-2$, then we fire the subgraph $\gamma_{g-i+1} \cup \gamma_{g-i+2} \cup \cdots \cup \gamma_{g}$ in two steps, first eliminating the negative chip, and second moving all of the remaining chips off of vertex $v_{g-i}$. We thus compute $Z_{g-i-1}=Z_{g-i}$ (no increase) and $Y_{g-i}=Z_{g-i}-\underline{x}_{g-i}-2$. 
Following this process of $v_{0}$-reduction, it is clear that the tuple representing the $v_{0^{-}}$ reduction of $K-c$ is $\left(s ; Y_{1}, Y_{2}, \ldots Y_{g-1}, Y_{g}\right)$. Furthermore, as we see from the above three cases, corresponding to firing $\gamma_{g-i+1} \cup \gamma_{g-i+2} \cup \cdots \cup \gamma_{g}$, we have

$$
\underline{x}_{g-i}+Y_{g-i}=Z_{g-i-1}-2 \text { for all } 1 \leqslant i \leqslant g-1 .
$$

We therefore finish the proof by proving simultaneously $Z_{g-i-1}=z_{g-i-1}$ and $Y_{g-i}=\underline{y}_{g-i}$, for all $0 \leqslant i \leqslant g-1$, by double-induction.

Note that the base cases $z_{g-1}=Z_{g-1}=2, z_{g-2}=Z_{g-2}=3, \underline{y}_{g}=Y_{g}=0$ and $\underline{y}_{g-1}=Y_{g-1}=1-\underline{x}_{g-1}$ were shown above. Assume now by induction that $z_{g-i}=Z_{g-i}$ and $\underline{y}_{g-i+1}=Y_{g-i+1}$. If the entry $i$ is in the last column or last row of $T$, then $z_{g-i-1}=z_{g-i}+1$ by definition, and $\underline{x}_{g-i}=0$ or $\underline{x}_{g-i}=z_{g-i}-1=Z_{g-i}-1$ by Lemma 42 and the induction hypothesis. It follows by cases (i) and (ii) above and the induction hypothesis again that $Z_{g-i-1}=Z_{g-i}+1=z_{g-i}+1=z_{g-i-1}$ in these cases.

On the other hand, if the entry $i$ is not in the last row or last column of $T$, then $z_{g-i-1}=z_{g-i}$ by definition, and $0<\underline{x}_{g-i}<Z_{g-i}-2$ by Lemma 42 . Then by case (iii), we have $Z_{g-i-1}=Z_{g-i}$ and the equality $z_{g-i-1}=Z_{g-i-1}$ follows again by induction.

Finally, in both cases, the identities (6) and (7) imply the equality of $\underline{y}_{g-i}$ and $Y_{g-i}$, thus finishing this inductive proof.

Example 43. Let $c=\phi(T)$ denote the chip-configuration $(2 ; \underline{2}, \underline{3}, \underline{1}, \underline{0}, \underline{1}, \underline{0})$, i.e. the running example, Example 7, where $T$ is the tableau

$$
T=\begin{array}{|l|l|l|}
\hline 1 & 3 & 4 \\
\hline 2 & 5 & 6 \\
\hline
\end{array} .
$$

By successively firing (i) $\gamma_{6}$, (ii) $\gamma_{5} \cup \gamma_{6}$, (iii) $\gamma_{4} \cup \gamma_{5} \cup \gamma_{6}$, (iv) $\gamma_{4} \cup \gamma_{5} \cup \gamma_{6}$ again, (v) $\gamma_{3} \cup \cdots \cup \gamma_{6}$, (vi) $\gamma_{2} \cup \cdots \cup \gamma_{6}$, and finally (vii) $\gamma_{2} \cup \cdots \cup \gamma_{6}$ again, we $v_{0}$-reduce the divisor $K-c$ into the divisor $(1 ; \underline{1}, \underline{0}, \underline{1}, \underline{2}, \underline{0}, \underline{0})$. See Figure 8. Using formula (3) to compute $\phi\left(T^{t}\right)$, where

$$
T^{t}=\begin{array}{|l|l|}
\hline 1 & 2 \\
\hline 3 & 5 \\
\hline 4 & 6 \\
\hline
\end{array}
$$

we do indeed obtain that $\phi\left(T^{t}\right)$ is the $v_{0}$-reduced divisor equivalent to $K-\phi(T)$.

\section{Acknowledgments}

This research was conducted at the 2011 and 2012 summer REU (Research Experience for Undergraduates) programs at the University of Minnesota, Twin Cities, and was partially funded by NSF grants DMS-1001933, DMS-1067183, and DMS-1148634. This led to an REU report [1] predating this article. The authors would like to thank Profs. Vic Reiner and Pavlo Pylyavskyy, who along with author Gregg Musiker directed the program, for their support. We would also like to thank the anonymous referees for their comments and suggestions. 

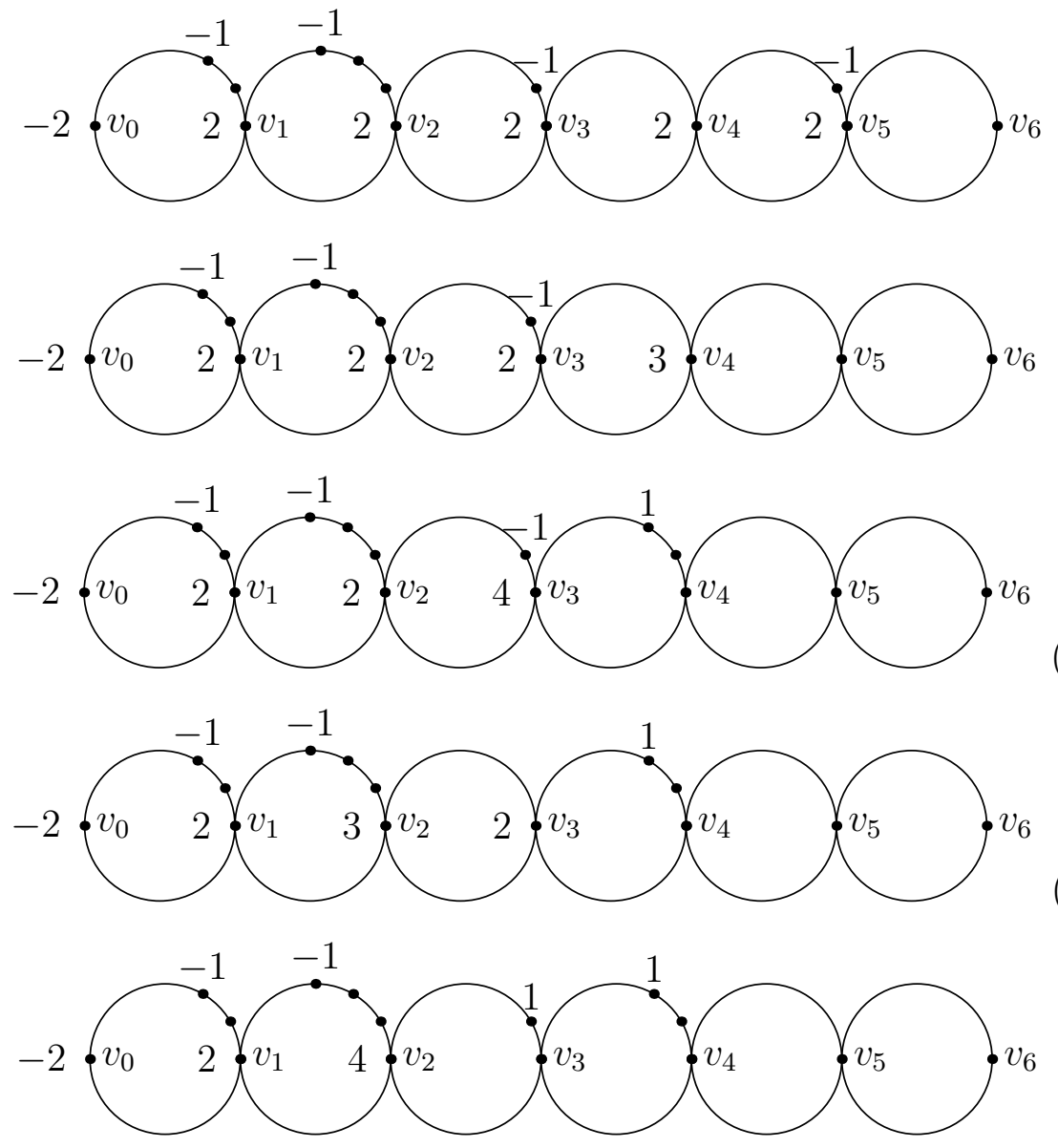

( v)
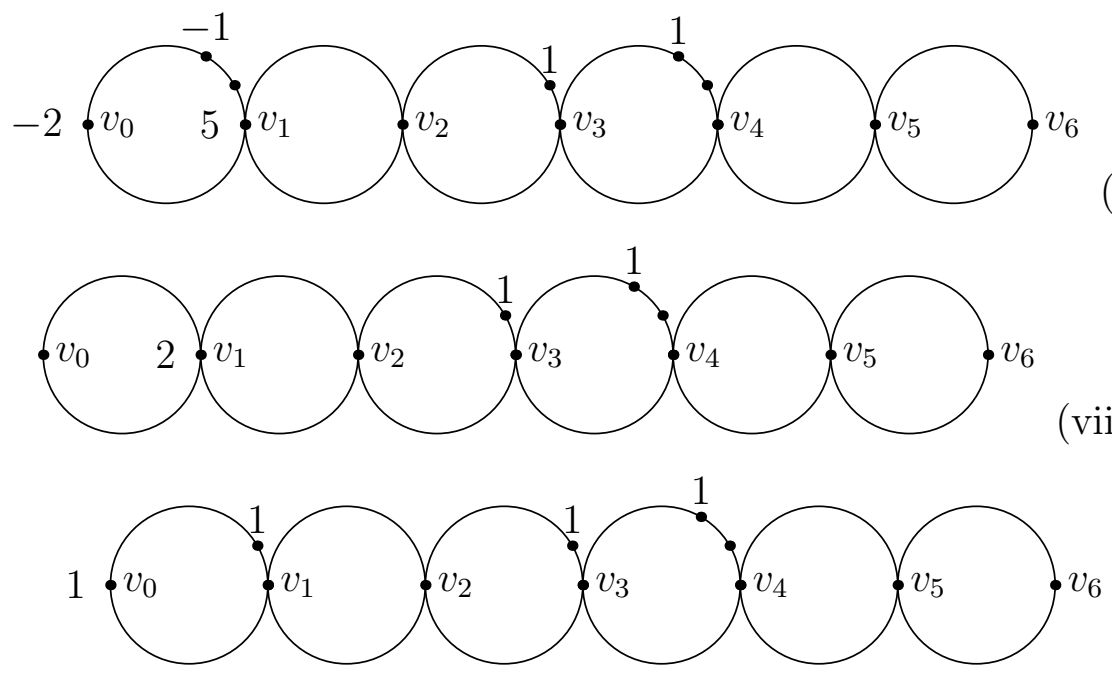

Figure 8: Successively $v_{0}$-reducing the divisor $(K-c)$ to $\phi\left(T^{t}\right)$ 


\section{References}

[1] Rohit Agrawal, Vladmir Sotirov, and Fan Wei. Evacuation of standard young tableaux and chip-firing. http://math.umn.edu/ ${ }^{\sim}$ reiner/REU/ AgrawalSotirovWei2011.pdf, June 2011.

[2] M. Baker and X. Faber. Metrized graphs, laplacian operators, and electrical networks. Contemporary Mathematics, 415:15-34, 2006.

[3] M. Baker and S. Norine. Riemann-Roch and Abel-Jacobi theory on a finite graph. Advances in Mathematics, 215(2):766-788, 2007.

[4] Matthew Baker. Specialization of linear systems from curves to graphs. Algebra $\&$ Number Theory, 2(6):613-653, Oct 2008.

[5] Filip Cools, Jan Draisma, Sam Payne, and Elina Robeva. A tropical proof of the Brill-Noether theorem. Advances in Mathematics, 230(2):759 - 776, 2012.

[6] D. Dhar. Self-organized critical state of sandpile automaton models. Physical Review Letters, 64(14):1613-1616, 1990.

[7] A. Gathmann and M. Kerber. A riemann-roch theorem in tropical geometry. Mathematische Zeitschrift, 259(1):217-230, 2008.

[8] Christian Haase, Gregg Musiker, and Josephine Yu. Linear systems on tropical curves. Mathematische Zeitschrift, 270:1111-1140, 2012.

[9] Ye Luo. Rank-determining sets of metric graphs. Journal of Combinatorial Theory Series A, 118(6):1775-1793, 2011.

[10] Grigory Mikhalkin and Ilia Zharkov. Tropical curves, their Jacobians and theta functions. In Curves and abelian varieties, volume 465 of Contemp. Math., pages 203-230. Amer. Math. Soc., Providence, RI, 2008.

[11] Sam Payne. Personal communication. 2012.

[12] Alexander Postnikov and Boris Shapiro. Trees, parking functions, syzygies, and deformations of monomial ideals. Transactions of the American Mathematical Society, 356(8):3109-3142, 2004.

[13] MP Schützenberger. Evacuations. Colloquio Internazionale sulle Teorie Combinatorie (Rome, 1973), 1:257-264, 1976.

[14] Richard Stanley. Promotion and evacuation. The Electronic Journal of Combinatorics, 16(2):R9, 2008. 